\title{
ANESTESIA TÓPICA MÁS SUBCONJUNTIVAL VERSUS RETROBULBAR EN LA ESCLERECTOMÍA PROFUNDA NO PENETRANTE
}

\section{COMBINED TOPICAL PLUS SUBCONJUNCTIVAL ANESTHESIA VERSUS RETROBULBAR ANESTHESIA IN NON-PENETRATING SCLERECTOMY}

\author{
GUTIÉRREZ-ORTIZ C ${ }^{1}$, TEUS M${ }^{1}$, CASTRO M², BOLÍVAR G ${ }^{2}$, CASTEJÓN M²
}

\section{RESUMEN}

Objetivo: Comparar la eficacia y seguridad de la anestesia tópica-subconjuntival con la retrobulbar en la escleroctomía profunda no penetrante suplementada con 5-FU.

Método: Se realizó un estudio prospectivo y aleatorizado de 30 pacientes consecutivos a los que se les practicó anestesia tópica-subconjuntival $(\mathrm{n}=14)$ o retrobulbar $(n=16)$. Se evaluaron las condiciones operatorias, el confort del paciente, el dolor postoperatorio, el dolor total y el resultado quirúrgico en cuanto a bajada de presión intraocular.

Resultados: No hubo diferencias en las condiciones quirúrgicas entre ambos grupos. El grupo con anestesia retrobulbar refirió más dolor durante la aplicación del anestésico $(P=0,00)$. El grupo con anestesia tópica-subconjuntival refirió más disconfort intraoperatoriamente $(\mathrm{P}=0,00)$. Sin embargo, esto no representó un problema para el cirujano. No hubo diferencias en el dolor postoperatorio entre ambos grupos. No hubo diferencias estadísticamente significativas en cuanto al dolor total. No hubo

\begin{abstract}
Purpose: To compare the safety and efficacy of topical plus subconjunctival versus retrobulbar anesthesia for primary non-penetrating sclerectomy supplemented with adjuntive 5-FU.

Methods: A prospective study of 30 consecutive patients who were randomized to receive subconjunctival $(n=14)$ or retrobulbar $(n=16)$ anesthesia was performed. Operating conditions, patient comfort, postoperative pain, total pain and surgical outcomes were evaluated.

Results: There were no differences in the operating conditions. The retrobulbar group reported significantly more pain during administration of the anesthetic agent than the subconjunctival group $(\mathrm{P}=$ 0.00 ). The subconjunctival group reported more discomfort during surgery than the retrobulbar group $(\mathrm{P}=0.00)$; however, this feature was not a problem for the surgeon. No statistically significant differences were found in regard to the total pain experienced, the postoperative pain, nor the success rates of the operative procedure in either group.
\end{abstract}

\footnotetext{
Recibido: 31/5/06. Aceptado: 18/4/07.

Hospital Universitario Príncipe de Asturias. Alcalá de Henares. Madrid. España.

1 Doctor en Medicina.

2 Licenciado en Medicina.

Correspondencia:

Consuelo Gutiérrez

C/. Mercurio, $63,2 .^{\circ} \mathrm{B}$

28032 Madrid

España

E-mail: consuelogutierre@ hotmail.com
} 
diferencia en cuanto al éxito quirúrgico entre ambos grupos.

Conclusiones: La anestesia tópica-subconjuntival es una alternativa segura y efectiva para la escleroctomía no penetrante suplementada con 5-FU.

Palabras clave: Anestesia, escleroctomía profunda no penetrante, cirugía glaucoma, tópica, retrobulbar, subconjuntival.

\section{INTRODUCCIÓN}

La escleroctomía profunda no penetrante (EPNP) lleva siendo una de las técnicas más usadas para el tratamiento quirúrgico del glaucoma (1). Puede realizarse empleando varias formas de anestesia: desde la general, a la retrobulbar, hasta las técnicas menos invasivas como la subconjuntival o la tópica. Sin embargo, existen puntos controvertidos en cuanto a cuál podría ser la anestesia más idónea para los sujetos que van a someterse a una cirugía de glaucoma. Por una parte, se ha publicado que la tasa de éxito quirúrgico en la trabeculectomía es menor cuando se usa anestesia subconjuntival comparada con la retrobulbar (2) y por otra, se ha observado que la retrobulbar induce una gran reducción de velocidad de flujo en los vasos retrobulbares en contraste con la anestesia subconjuntival. Por tanto, podría inferirse que la técnica subconjuntival sería preferible en sujetos con problemas de perfusión ocular como los pacientes con glaucoma (3) ya que, teóricamente, no reduciría el flujo de estos vasos. Además la anestesia tópica-subconjuntival evitaría complicaciones como la perforación del globo o la depresión respiratoria (4).
Conclusion: Topical/subconjunctival anesthesia is a safe and effective alternative to retrobulbar anesthesia for non penetrating sclerectomy supplemented with 5-FU (Arch Soc Esp Oftalmol 2007; 82: 285-290).

Key words: Anesthesia, non-penetrating sclerectomy, glaucoma surgery, topical, retrobulbar, subconjunctival.

Tabla I. Características demográficas de los pacientes del estudio

\begin{tabular}{lccc}
\hline Parámetro & $\begin{array}{c}\text { Grupo de anestesia } \\
\text { tópica-subconjuntival }\end{array}$ & $\begin{array}{c}\text { Grupo de anestesia } \\
\text { retrobulbar }\end{array}$ & $P$ \\
\hline $\begin{array}{l}\text { Número de ojos } \\
\text { Edad (años) }\end{array}$ & 14 & 16 & 63 DE: 10,4 \\
Sexo & $\begin{array}{c}\text { (rango: } 46-76-72) \\
\text { (rango: } 48-78)\end{array}$ & 0,33 \\
$\quad \begin{array}{c}\text { Mujeres } \\
\text { Hombres }\end{array}$ & 6 & 9 & 0,71 \\
$\begin{array}{l}\text { Glaucoma primario de ángulo abierto } \\
\text { Agudeza visual preoperatoria }\end{array}$ & 8 & 7 & \\
PIO preoperatoria (mmHg) & 0,7 DE: 0,22 & 0,63 DE: 0,34 & 0,11 \\
& (rango: $0,2-1)$ & (rango: cd-1) & 0,11 \\
\hline \hline
\end{tabular}


$(\leq 21 \mathrm{~mm} \mathrm{Hg}$ en defectos leves, $\leq 17 \mathrm{~mm} \mathrm{Hg}$ en defectos moderados, $\leq 15 \mathrm{~mm} \mathrm{Hg}$ en defectos severos), progresión campimétrica del defecto a pesar de tratamiento médico máximo, pacientes no cumplidores o intolerancia a tratamiento hipotensor. El grosor corneal fue tomado en cuenta como factor de riesgo.

Los criterios de exclusión fueron los siguientes: cirugía de glaucoma previa, historia de alergia a anestésicos, ansiedad excesiva, demencia, hipoacusia o poca capacidad de fijación por estrabismo o nistagmus.

Después de haber sido informados del procedimiento todos los pacientes firmaron el correspondiente consentimiento aprobado por el comité ético del hospital. El estudio se llevó a cabo cumpliendo los criterios de la declaración de Helsinki.

Los pacientes fueron aleatoriamente asignados a anestesia tópica más subconjuntival o retrobulbar mediante una tabla generada por ordenador.

Los pacientes en el grupo tópica-subconjuntival recibieron una gota de lidocaína al 5\%, 5 y 10 minutos antes de la cirugía. Los pacientes del grupo de anestesia retrobulbar recibieron una inyección de 5 $\mathrm{ml}$ de mezcla de bupivacaína y mepivacaína al $50 \%$. No se utilizó sedación en ninguno de los grupos. Al grupo de anestesia tópica-subconjuntival, antes de iniciar la cirugía, se les inyectó $0,5 \mathrm{ml}$ de lidocaína al $1 \%$ a las $12 \mathrm{~h}$ ya bajo el microscopio quirúrgico.

En ambos grupos la técnica quirúrgica fue la misma. Se realizó un punto de tracción corneal con vycril de 7/0 para conseguir una buena visualización del área. Se realizó el flap conjuntival con base fórnix. Se cauterizó la zona elegida con diatermia bipolar y a continuación se aplicó una esponja quirúrgica embebida en 5-Fluorouracilo $50 \mathrm{mg} / \mathrm{ml}$ durante 5 minutos bajo la conjuntiva. Posteriormente, se talló el primer colgajo escleral de $5 \times 5 \mathrm{~mm}$ y a continuación el colgajo profundo de 4x4 mm. Cuando se llegó a la membrana trabéculo descemética se peló el suelo del canal de Schlemm y se realizó el corte del colgajo profundo. No se aplicó ningún tipo de implante. El flap escleral se suturó con dos puntos sueltos de Nylon 10/0 en los extremos y la conjuntiva con puntos sueltos de Nylon 10/0.

Una hora tras la cirugía los pacientes fueron interrogados por un observador independiente sobre el grado de dolor sentido durante la anestesia, durante la cirugía y tras ella, con una escala de 10 puntos desde 0 para no dolor hasta 10 para un dolor extremo. El cirujano también rellenó dos cuestionarios sobre confort durante la cirugía con una escala de 0 a 5 con 0 para no problemas y 5 para problemas graves y grado de colaboración del paciente con una escala de 0 a 5 donde 0 sería no colaboración y 5 una colaboración excelente.

Sólo aquellos pacientes que pudieron ser seguidos durante al menos 3 meses fueron incluidos en el estudio.

Se definió éxito quirúrgico si la cirugía consiguió disminuir la presión intraocular al menos un $30 \%$ con respecto a la $\mathrm{PIO}$ preoperatoria con tratamiento y ésta no descendió por debajo de $6 \mathrm{mmHg}$.

Se revisó a los pacientes al día siguiente de la cirugía, a la semana, y tras 1, 3, 6, 9 y 12 meses. Se realizaron visitas adicionales si así lo consideró oportuno el cirujano.

El análisis estadístico se realizó con el programa SPSS 12.0 para Windows (SPSS Inc, Chicago, Illinois, USA). Se usaron los test de Mann-Whitney y chi-cuadrado o test exacto de Fisher. Se consideró significación estadística si $\mathrm{P}<0,05$.

\section{RESULTADOS}

Se estudiaron 30 ojos de 30 pacientes, 14 fueron operados bajo anestesia tópica-subconjuntival y 16 bajo anestesia retrobulbar. El tiempo medio de seguimiento fue de 8 DE: 0,65 meses (rango: 3-12 meses). La tabla I muestra los datos demográficos de los dos grupos.

Los grupos de anestesia tópica-subconjuntival y el grupo de anestesia retrobulbar no mostraron diferencias estadísticamente significativas en cuanto PIO preoperatorio, PIO postoperatoria, condiciones de la cirugía o grado de colaboración. Sí se obtuvieron diferencias estadísticamente significativas en cuanto al grado de dolor durante la anestesia y durante la cirugía (tabla II y fig. 1). En todos los casos incluidos en el grupo de anestesia tópica la cirugía se realizó sin cambiar el tipo de anestesia inicialmente planeado. Tres pacientes del grupo de anestesia retrobulbar requirieron la aplicación de suplemento tópico con lidocaína al $5 \%$.

No se registraron complicaciones en ninguno de los dos grupos. No se observó hemorragia subconjuntival durante el procedimiento en el grupo de anestesia tópica. En un paciente del grupo retrobulbar hubo quemosis conjuntival hemorrágica que no dificultó la cirugía. Los movimientos inadvertidos fueron más frecuentes en el grupo de anestesia tópi- 
Tabla II. Presiones intraoculares, éxito quirúrgico, dolor y grado de colaboración de ambos grupos a estudio

\begin{tabular}{|c|c|c|c|}
\hline Parámetro & $\begin{array}{l}\text { Grupo de anestesia } \\
\text { tópica-subconjuntival }\end{array}$ & $\begin{array}{l}\text { Grupo de anestesia } \\
\text { retrobulbar }\end{array}$ & $\mathrm{P}$ \\
\hline PIO postoperatoria al final del seguimiento (mm Hg) & $\begin{array}{l}\text { 15,22 DE: } 3,89 \\
\text { (rango: } 6-21 \text { ) }\end{array}$ & $\begin{array}{l}14 \mathrm{DE}: 4,16 \\
\text { (rango: } 6-24 \text { ) }\end{array}$ & 0,18 \\
\hline Éxito & 12 & 13 & 0,68 \\
\hline Número de fármacos preoperatorios & $\begin{array}{l}\text { 1,71 DE: } 0,6 \\
\text { (rango } 1-3 \text { ) }\end{array}$ & $\begin{array}{l}\text { 1,88 DE: } 0,95 \\
\text { (rango } 1-4 \text { ) }\end{array}$ & 0,11 \\
\hline Número de fármacos al final del seguimiento & $\begin{array}{l}\text { 0,12 DE: } 0,35 \\
\text { (rango: } 0-1 \text { ) }\end{array}$ & $\begin{array}{l}\text { 0,06 DE: } 0,25 \\
\text { (rango: } 0-1 \text { ) }\end{array}$ & 0,56 \\
\hline Condiciones de la cirugía $(0-5)$ & $\begin{array}{l}\text { 4,9 DE: } 0,3 \\
\text { (rango: } 4-5 \text { ) }\end{array}$ & $\begin{array}{c}5 \mathrm{DE}: 0,00 \\
\text { rango }(5-5)\end{array}$ & 0,52 \\
\hline Grado colaboración paciente $(0-5)$ & $\begin{array}{l}4,9 \mathrm{DE}: 0,3 \\
\text { (rango: } 4-5 \text { ) }\end{array}$ & $\begin{array}{l}\text { 4,93 DE: } 0,25 \\
\text { (rango: } 4-5 \text { ) }\end{array}$ & 0,78 \\
\hline Dolor subjetivo del paciente durante la anestesia (0-10) & $\begin{array}{l}0,54 \mathrm{DE}: 0,93 \\
\text { (rango: } 0-3 \text { ) }\end{array}$ & $\begin{array}{l}\text { 4,25 DE: } 2,1 \\
\text { (rango: } 3-10 \text { ) }\end{array}$ & 0,00 \\
\hline Dolor subjetivo del paciente durante la cirugía (0-10) & $\begin{array}{l}2,27 \text { DE: } 1,4 \\
\text { (rango: } 0-5 \text { ) }\end{array}$ & $\begin{array}{l}0,43 \mathrm{DE}: 0,62 \\
\text { (rango: } 0-2 \text { ) }\end{array}$ & 0,00 \\
\hline Dolor subjetivo del paciente tras la cirugía $(0-10)$ & $\begin{array}{l}0,27 \text { DE: } 0,46 \\
\text { (rango: } 0-1 \text { ) }\end{array}$ & $\begin{array}{c}0,27 \text { DE } 0,46 \\
\text { (rango: } 0-1 \text { ) }\end{array}$ & 0,99 \\
\hline Dolor total $(0-10)$ & $\begin{array}{l}3,09 \text { DE: } 1,75 \\
\text { (rango: } 1-7 \text { ) }\end{array}$ & $\begin{array}{l}\text { 5,12 DE: } 2.91 \\
\text { (rango: } 3-13 \text { ) }\end{array}$ & 0,20 \\
\hline
\end{tabular}

ca-subconjuntival $(\mathrm{P}=0,003)$ sin embargo, estos movimientos no representaron un problema para realizar la cirugía y no llevaron a ningún tipo de complicación.

En cuanto a la eficacia del procedimiento se obtuvo un éxito quirúrgico en $12(81,25 \%)$ pacientes en el grupo de anestesia tópica-subconjuntival y en $13(85,71 \%)$ de los pacientes en el grupo de anestesia retrobulbar. No hubo diferencias estadísticamente significativas entre los dos grupos a estudio en cuanto al éxito quirúrgico $(\mathrm{P}=0,68)$. Las presiones al final del seguimiento fueron de 15,22 DE: 3,89 en el grupo de anestesia tópica y de $14 \mathrm{DE}$ : 4,16 en el grupo de anestesia retrobulbar (fig. 2). Estas diferencias tampoco resultaron estadísticamente significativas $(\mathrm{P}=0,18)$ entre ambos grupos. $\mathrm{El}$ número de fármacos postoperatorios en el grupo de anestesia tópica-subconjuntival fue de 0,12 DE: 0,35 y de 0,06 DE: 0,25 en el grupo de anestesia

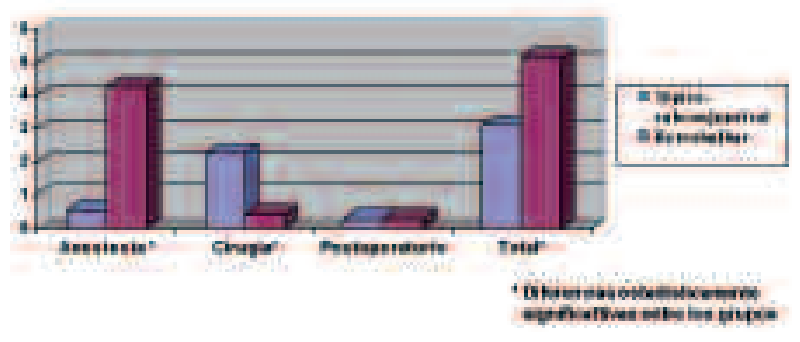

Fig. 1: Dolor perioperatorio. retrobulbar, estas diferencias tampoco alcanzaron significación estadística $(\mathrm{P}=0,56)$ entre ambos grupos.

\section{DISCUSIÓN}

Este estudio muestra que la EPNP realizada con anestesia tópica-subconjuntival es una técnica confortable tanto para el paciente como para el cirujano e igualmente efectiva que si se realiza con anestesia retrobulbar.

En primer lugar, el grado de dolor sentido durante la aplicación del anestésico es significativamente menor que cuando se administra el anestésico retrobulbarmente. Por otra parte, el grupo de anestesia retrobulbar presenta de forma estadísticamente significativa menor dolor durante la cirugía. Sin

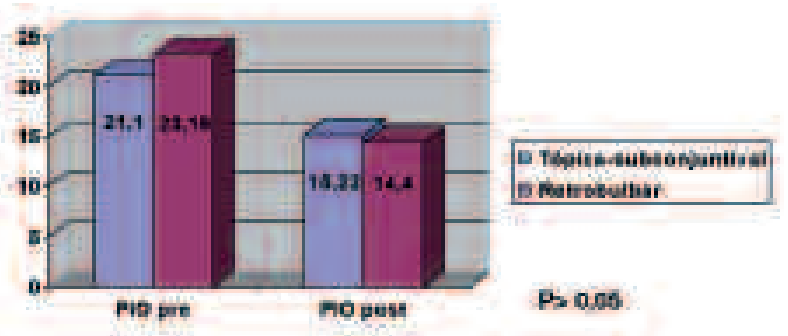

Fig. 2: Presiones intraoculares en ambos grupos antes y después de la cirugía. 
embargo, ambos grupos presentan el mismo dolor posquirúrgico. Si analizamos el dolor total, éste es ligeramente menor en el grupo de anestesia tópica, aunque las diferencias no alcanzan significación estadística. Por lo que pensamos que es una técnica confortable para el paciente. No se obtuvieron diferencias estadísticamente significativas en cuanto al confort del cirujano y al grado de colaboración del paciente. Con anestesia tópica-subconjuntival el movimiento ocular se mantiene, lo que, en ocasiones mejora las condiciones optimizando el acceso quirúrgico. Sin embargo, el mayor disconfort sentido por el paciente puede disminuir ligeramente estas condiciones por movimientos involuntarios o por la necesidad de suplementar con anestésico tópico, sin embargo, esto también puede suceder con la anestesia retrobulbar. Nuestros resultados se ven avalados por otros trabajos (6).

La trabeculectomía se ha realizado bajo diferentes técnicas anestésicas entre ellas la subconjuntival. Edmus en 2004 publicó que la anestesia subconjuntival obtiene malos resultados en cuanto a reducción tensional ya que puede causar hemorragia y daño en los tejidos y así estimular los fibroblastos (2). Sin embargo, sus resultados no concuerdan con los nuestros ni con los de otros autores $(7,8)$. En nuestro estudio no se produjo ningún caso de hemorragia subconjuntival durante la inyección de la anestesia subconjuntival, y sí apareció quemosis hemorrágica en un caso en los que aplicamos la técnica retrobulbar. El estudio de Edmuns es observacional y cuenta con cirujanos no experimentados con la trabeculectomía por lo que los resultados pueden estar sesgados. Por otra parte, Edmuns analiza la trabeculectomía y en nuestro estudio analizamos la esclerectomía profunda no penetrante suplementada con 5-FU donde puede que los procesos de cicatrización subconjuntival no se desarrollen de la misma manera. Por otra parte, nuestro estudio concuerda con los resultados obtenidos con AzuaraBlanco y colaboradores (7) quienes encontraron que la anestesia subconjuntival era una alternativa efectiva a la anestesia retrobulbar. Por otro lado, los resultados de este estudio concuerdan con los publicados por Noureddin y sus colaboradores quienes encontraron que los pacientes operados con lidocaína subconjuntival tenían una PIO postoperatoria menor que aquellos que se habían sometido a anestesia general (8).

Nuestro trabajo no ha demostrado diferencias estadísticamente significativas en cuanto a la reduc- ción de PIO en ambos grupos, lo cual podría deberse al pequeño tamaño muestral. Por tanto, se necesitan estudios prospectivos con mayor número de pacientes para apoyar los resultados obtenidos en nuestro estudio.

Por otra parte, se ha observado que la técnica retrobulbar induce una gran reducción de velocidad de flujo en los vasos retrobulbares en contraste con la anestesia subconjuntival. Por tanto, la técnica subconjuntival sería preferible en pacientes con problemas de perfusión ocular como los pacientes con glaucoma (3) en los que una reducción adicional de la velocidad de flujo en los vasos durante la anestesia podría tener un efecto deletéreo en su función visual.

Estudios realizados por Naor sugieren que la anestesia tópica-subconjuntival evita complicaciones tales como la hemorragia retrobulbar, o la inyección intravascular o intratecal, así como la perforación del globo y la depresión respiratoria (4). Además la anestesia subconjuntival administrada en el lugar planeado para la escleroctomía tendría ventajas adicionales sobre la retrobulbar ya que permitiría una disección roma de los tejidos. Por otra parte, en pacientes con campos visuales reducidos o mala agudeza visual, la no necesidad de ocluir el ojo tras la cirugía podría ser una ventaja añadida para el paciente.

La anestesia tópica-subconjuntival para la EPNP suplementada con 5-FU es una técnica segura y confortable tanto para los pacientes como para los cirujanos experimentados con la cirugía no penetrante de glaucoma. Así mismo, se consiguen similares controles tensionales a medio plazo que con la anestesia retrobulbar y puede resultar especialmente útil en pacientes con campos visuales muy reducidos o mala agudeza visual. Sin embargo, solo sería recomendable para cirujanos experimentados en esta técnica quirúrgica para evitar sangrados subconjuntivales que pudieran inducir aumento de la cicatrización o complicaciones por movimientos inadvertidos del paciente.

\section{BIBLIOGRAFÍA}

1. Lachkar $Y$, Hamard P. Nonpenetrating filtering surgery. Curr Opin Ophthalmol 2002; 13: 110-115.

2. Edmuns B, Bunce CV, Thompson JR, Salmon JF, Wormald RP. Factors associated with success in first-time trabeculectomy for patients at low risk of failure with chronic open-angle glaucoma. Ophthalmology 2004; 111: 97-103. 
3. Huber KK, Remky A. Effect of retrobulbar versus subconjunctival anaesthesia on retrobulbar haemodynamics. Br J Ophthalmol 2005; 89: 719-723.

4. Naor J, Slomovic AR. Anesthesia modalities for cataract surgery. Curr Opin Ophthalmol 2000; 11: 7-11.

5. Advanced Glaucoma Intervention Study. 2. Visual field test scoring and reliability. Ophthalmology 1994; 101: 1445-1455.

6. Vicary D, McLennan S, Sun XY. Topical plus subconjunc- tival anesthesia for phacotrabeculectomy: one year follow-up. J Cataract Refract Surg 1998; 24: 1247-1251.

7. Azuara-Blanco A, Moster MR, Marr BP. Subconjunctival versus peribulbar anesthesia in trabeculectomy: a prospective, randomized study. Ophthalmic Surg Lasers 1997; 28: 896-899.

8. Noureddin BN, Jeffrey M, Franks WA, Hitchings RA. Conjunctival changes after subconjunctival lignocaine. Eye1993; 7: 457-460. 